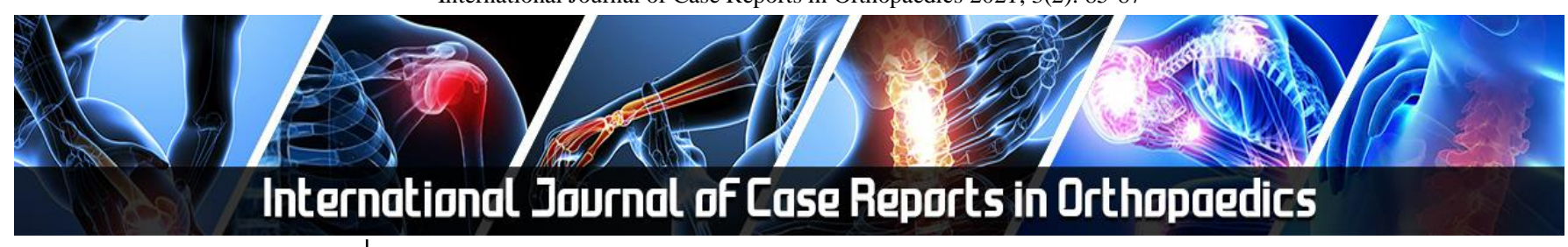

E-ISSN: 2707-8353 P-ISSN: 2707-8345 IJCRO 2021; 3(2): 85-87 Received: 08-05-2021 Accepted: 10-06-2021

\section{Tim Truyers}

Department of Orthopaedic Surgery, Zuyderland Medical Centre, Henri Dunantstraat 5 , 6419 PC Heerlen, Netherlands

\section{Remco Meijer}

Department of Orthopaedic Surgery, Zuyderland Medical Centre, Henri Dunantstraat 5 , 6419 PC Heerlen, Netherlands

\section{Ralf Walbeehm}

Department of Orthopaedic Surgery, Zuyderland Medical Centre, Henri Dunantstraat 5, 6419 PC Heerlen, Netherlands

Corresponding Author: Tim Truyers

Department of Orthopaedic Surgery, Zuyderland Medical Centre, Henri Dunantstraat 5, 6419 PC Heerlen, Netherlands

\section{Fracture of the capitellum with an associated fracture of the lateral humeral condyle: A case report}

\author{
Tim Truyers, Remco Meijer and Ralf Walbeehm
}

DOI: https://doi.org/10.22271/27078345.2021.v3.i2b.71

\begin{abstract}
Isolated fractures of the capitellum humeri are rare and can be difficult to diagnose. Associated bone and soft tissue injuries are common. Mostly, a concomitant lateral collateral ligament injury or a radial head fracture is reported. Open reduction and internal fixation are accepted as the favored treatment to facilitate anatomic reduction. Missed diagnosis or inadequate treatment of capitellar fractures can led to the development of advanced degenerative changes, limitation of motion and pain. We present the case of an eighteen-year-old male with a Bryan and Morrey type I capitellar fracture in association with a fracture of the lateral humeral condyle. Fractures were treated with open reduction and internal fixation, using a headless compression screw and cannulated screws. To our knowledge this is the first case report to describe the treatment of a capitellar fracture with an associated fracture of the lateral humeral condyle.
\end{abstract}

Keywords: Distal humerus, capitellum, capitellar fracture, shear fracture, lateral humeral condyle fracture

\section{Introduction}

Capitellar fractures are relatively uncommon, accounting for $1 \%$ of all elbow fractures and $6 \%$ of fractures of the distal humerus ${ }^{[1-8]}$. Due to a more frequent occurrence of cubitus valgus or cubitus recurvatum the incidence is higher in the female population ${ }^{[4]}$. In 1853 , Hahn was the first one to describe a fracture of the capitellum ${ }^{[3]}$. Those fractures typically result from two traumatic mechanisms. A fall on an outstretched hand, with varying degrees of elbow flexion, generates an axial force through the radial head to the capitellum. The center of rotation of the capitellum lies $12-15 \mathrm{~mm}$ anterior to the humeral shaft and is therefore vulnerable to shearing fractures in the coronal plane. Or capitellar fractures can result from a spontaneous reduction after a posterolateral elbow subluxation or dislocation. The capitellum is then sheared off by the radial head or coronoid process ${ }^{[1,3,4,8]}$.

Several classification systems have been described. The Bryan and Morrey classification (modified by McKee et al.) distinguishes 4 types of fractures. Type 1 fractures (HahnSteinthal fractures) involve an isolated fracture of the capitellum with a significant amount of subchondral and cancellous bone of the lateral condyle; type 2 fractures (Kocher-Lorenz or Mouchet fractures) involve the articular cartilage overlying the capitellum with a subchondral shell; type 3 fractures are comminuted fractures of the capitellum. A type 4 fracture was later added, as the classification system was expanded by McKee et al. They consist of capitellar fractures extending into the trochlea. Dubberley et al. created a classification system providing information about the surgical management and prognosis of the fractures. Type 1 fractures are described as capitellar fractures with or without extension to the lateral trochlear ridge; type 2 fractures involve to capitellum and trochlea as one single fragment; and type 3 fractures involve the capitellum and trochlea splitted into two fragments. In case of presence of posterior condylar comminution fractures are further classified with a $B$, in case of absence fractures are denoted with an $A^{[1,2,3,8]}$.

The complexity of capitellar fractures is often underestimated on conventional plain radiographs. Therefore, an additional computed tomography (CT) can be useful to indent if the extend of the fracture and to plan operative treatment ${ }^{[1,3]}$.

In this case report, we present the case of an eighteen-year-old man with a Bryan and Morrey type I capitellar fracture in association with a fracture of the lateral humeral condyle. Fractures were treated with open reduction and internal fixation.

The patient was informed that data concerning the case would be submitted for publication. Consent was obtained. 


\section{Case report}

An eighteen-year-old man, with no relevant medical history, presented at the emergency department (ER) after sustaining a fall from a scooter onto an outstretched left arm. Immediately, the patient experienced pain at his left elbow and he was unable to fully extend his left arm. On presentation, physical examination revealed mild swelling of the left elbow without tenderness over the osseous structures of the distal humerus or proximal radius and ulna. Flexion and extension of the elbow were restricted due to pain. Pronation and supination were similar to the uninjured site and painless. No neurovascular compromise was observed.

Plain radiographic images suggested a fracture of the lateral humeral condyle. Additionally, on the lateral view a curvilinear bony fragment on the volar aspect of the distal humerus was seen (Fig. 1). For better visualization of the fracture, computed tomography (CT) of the elbow was performed. CT confirmed a fracture of the capitellar articular surface with presence of a concomitant fracture of the lateral humeral condyle (Fig. 2). The capitellar fracture can be classified as a type I fracture (Hahn-Steinthal fracture) according to the Bryan and Morrey classification, or as a type IA fracture according to the Dubberley classification.

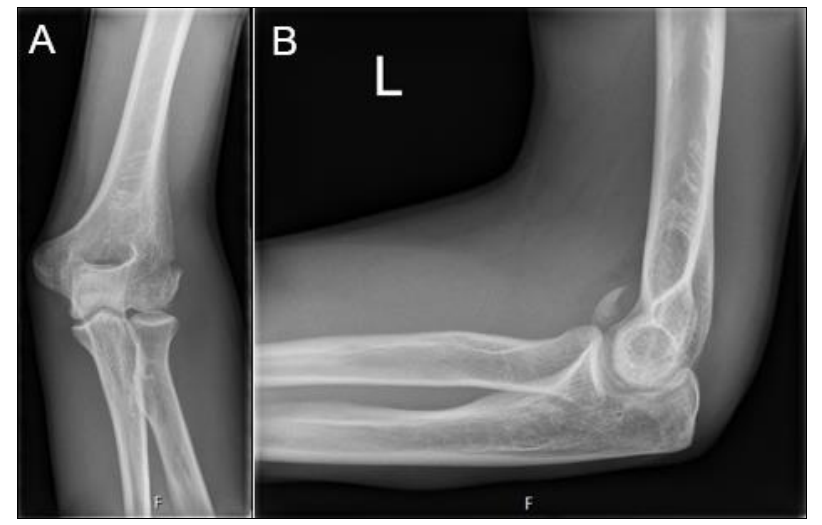

Fig 1: Pre-operative plain radiographs of the left elbow. (A) Anteroposterior radiograph suggests a fracture of the lateral humeral condyle. (B) Lateral radiograph shows a coronal shear fracture of the distal humerus.

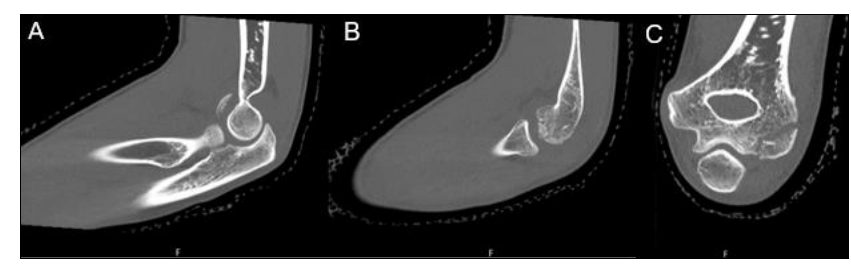

Fig 2: (A, B) Sagittal and (C) coronal computed tomography images of the left elbow showing a capitellar fracture with an associated fracture of the lateral humeral condyle.

After receiving informed consent from the patient, a surgical procedure was planned ten days after presentation at the ER. The patient received a long arm cast awaiting the operation. Surgery was performed under general and plexus anesthesia using a pneumatic tourniquet. Through a single lateral incision (Kaplan approach) the lateral condylar fracture and the capitellar fracture were identified. First, the capitellar fracture was reduced and temporary fixated by two K-wires. Definitive fixation was obtained by one Omnitech 2.0 screw $(14 \mathrm{~mm})$ placed in an anteroposterior direction. Secondly, the lateral condylar fracture was reduced and fixated with two De Puy Synthes cannulated screws 3.0 (34 and $50 \mathrm{~mm}$ ). The elbow was immobilized in a long arm cast for four weeks, followed by two weeks of non-weight bearing exercises.

At six weeks postoperatively, motion arc was $30^{\circ}$ to $110^{\circ}$ with unrestricted pronation and supination. At three months after surgery a full range of motion was regained. Motion arc was $0^{\circ}$ to $140^{\circ}$. Radiographs showed full consolidation of the fractures (Fig. 3).

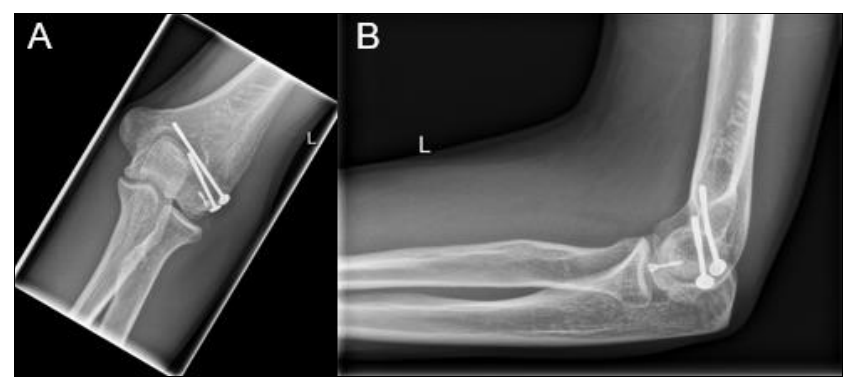

Fig 3: Plain radiographic images at three moths postoperatively.

(A) Anteroposterior and (B) lateral radiographs showing full consolidation of the fractures after open reduction and internal fixation, using a headless compression screw and two cannulated screws.

\section{Discussion}

However isolated fractures of the capitellum are rare, associated bone and soft tissue injuries are common. An incidence up to $60 \%$ of a concomitant lateral collateral ligament injury or a radial head fracture has been reported ${ }^{[1}$, 4]. Though the rarity of capitellar fractures data on associated injuries is limited. Raush et al. investigated the incidence of associated injuries by a retrospective study design. They found an associated elbow injury in $44 \%$ of the patients, in which a radial head fracture was the most common. There was no significant correlation between fracture type and kind of associated lesion ${ }^{[3]}$.

Some studies showed that associated injuries and fracture type affect the outcome after operative treatment. A metaanalysis performed by Shu-Kun He et al. showed significant higher MEPI (Mayo Elbow Performance Index) scores of patients without associated injuries after operative treatment. The arc of motion was not significantly different with presence of an associated lesion. Comparing the different fracture types, according to the Bryan and Morrey classification, no significant difference in MEPI score or range of motion was found. However, when applying the Dubberley classification system, patients with type $A$ fractures showed significant higher MEPI scores compared with patients with type $B$ fractures. Similar to the Bryan and Morrey classification, results showed no significant difference in range of motion between the fracture types ${ }^{[4]}$. Outcome after a non-operative treatment is often unpredictable ${ }^{[2,8]}$. Therefore, an operative treatment and especially open reduction and internal fixation are gradually accepted as the golden standard in the treatment of most capitellar fractures. The goal of a surgical treatment is to restore native anatomy and allow early elbow mobilization. A universally accepted method of surgical management has not been described yet ${ }^{[4,7,8]}$. A wide variety of techniques have been used to obtain a stable reduction, including $\mathrm{K}$ wires, biodegradable pins, headless compression screws, small fragment cancellous screws and plates ${ }^{[1]}$. In our case 
the capitellar fragment was fixated with one titanium headless compression screw in an anteroposterior direction. Additionally, the lateral condylar fracture was reduced and fixated with two cannulated screws. The study of Tanriverdi et al. showed some favorable results in outcome measurements after using headless compression screws in the treatment of capitellar fractures. They noted the development of avascular necrosis in $14 \%$ and heterotopic ossifications in $4 \%$ of the patients. Secondary elbow osteoarthritis was seen in $33 \%$ of the cases. This result was slightly higher than comparative literature. The authors stated this had been due to the inclusion of type 3 or comminuted fractures ${ }^{[6]}$.

A biomechanical study of Borbas et al. compared the biomechanical stability of three different internal fixation techniques for Dubberley type 1A fractures: two anteroposterior cannulated headless compression screws, two anteroposterior cannulated headless compression screws with an anterior antiglide plate and a posterolateral distal humeral locking plate. Results showed that fractures were biomechanically stabilized using the three different techniques. In view of the simplest and most cost-effective technique and the advantages of less metal ware, authors advised the use of two isolated anteroposterior headless compression screws ${ }^{[8]}$. To date, biodegradable implants are gaining importance. The Magnezix compression screw was developed in 2013 and is composed of the magnesium alloy magnesium-yttrium-rare earth- Zirconium (MgYREZr). The screws will be completely degraded after about one year. Mechanical properties are more similar to bone in comparison with steel or titanium implants. Wagner et al. conducted a biomechanical study comparing headless compression screws made from titanium or biodegradable magnesium. Testing was performed in a cadaveric model fixating Bryan and Morrey type 1 fractures. Results suggested that fractures treated with biodegradable headless compression screws of magnesium alloy showed similar biomechanical features to type 1 fractures fixated with titanium screws ${ }^{[7]}$.

\section{Conclusion}

The patient in our case obtained a full range of motion after operative treatment and was able to return to daily activities without any problems. As the importance of the capitellum has gained recognition, surgical techniques are evolving enormously. However, capitellar fractures are rare and literature is often based on limited data. Therefore, further research with larger study populations is required.

\section{References}

1. Carroll MJ, Athwal GS, King GJ, Faber KJ. Capitellar and Trochlear Fractures. Hand Clin 2015;31(4):615630.

2. Frank JM, Saltzman BM, Garbis N, Cohen MS. Articular shear injuries of the capitellum in adolescents. J Shoulder Elbow Surg 2016;25(9):1485-1490.

3. Rausch V, Königshausen M, Schildhauer TA, Gessmann J, Seybold D. Fractures of the capitellum humeri and their associated injuries. Obere Extrem 2018;13(1):33-37.

4. He SK, Xu L, Guo JH, Liao JP, Qin TW, Huang FG. The impact of associated injuries and fracture classifications on the treatment of capitellum and trochlea fractures: A systematic review and metaanalysis. Int J Surg 2018;54(Pt A):37-47.

5. Wolfson TS, Lowe D, Egol KA. Capitellum fracture open reduction and internal fixation with headless screws. J Orthop Trauma 2019;33(1):S5-S6.

6. Tanriverdi B, Kural C, Altun S. Capitellum fractures: Treatment with headless screws and outcomes. Jt Dis Relat Surg 2020;31(2):291-297.

7. Wagner FC, Post A, Yilmaz T, Maier D, Neubauer J, Feucht MJ et al. Biomechanical comparison of biodegradable magnesium screws and titanium screws for operative stabilization of displaced capitellar fractures. J Shoulder Elbow Surg 2020;29(9):19121919.

8. Borbas P, Vetter M, Loucas R, Hofstede S, Wieser K, Ernstbrunner L. Biomechanical stability of simple coronal shear fracture fixation of the capitellum. J Shoulder Elbow Surg 2021, S1058-2746(21)00057-4. 